\title{
Evidence for the persistence of visual guidance information
}

\author{
RICHARD A. TYRRELL, KIRSTEN K. RUDOLPH, BETH G. EGGERS, \\ and HERSCHEL W. LEIBOWITZ \\ Pennsylvania State University, University Park, Pennsylvania
}

\begin{abstract}
An earlier study from our laboratory provided initial support for the hypothesis that information facilitating visual guidance persists in the absence of retinal stimulation. The present study supports and extends this hypothesis with three experiments in which visually occluded subjects positioned a point of light at the location of a previously viewed target and also walked in the direction of a previously viewed path. In both tasks, performance was possible following occlusion, and in all cases, performance slowly and significantly decreased with longer durations of occlusion. This decay in performance was gradual and had a "half-life" of greater than 15 sec. Absolute performance was correlated across tasks. The effect of occlusion on absolute error in the localization performance was relatively stable within individuals over a 3-week period. The biological utility of guidance information persistence is discussed along with implications for space constancy, illusions of motion, and problems of disorientation.
\end{abstract}

With little or no effort, we are able to maintain an adequate sense of our position as we move through the environment. Without having to think about our position in space, we can attend even to demanding tasks as we move. Although we are most skilled at maneuvering through space when illumination is high, our ability to maneuver is nearly as skillful when illumination approaches absolute threshold levels. Indeed, common experience suggests that we are able to find our way through an environment we have recently seen even when there is no retinal information to guide our movements. Certainly some form of spatial memory supports this skill. However, little systematic research has been carried out regarding this form of memory, especially in comparison with the vast literature on other types of memory.

The ability to orient and locomote with reduced retinal information is further exemplified by the common activity of reading while walking. A substantial portion of the

\footnotetext{
Portions of this research were presented at the 1990 and 1991 meetings of the Association for Research in Vision and Ophthalmology, Sarasota, FL (Tyrrell, Rudolph, Eggers, Li, \& Leibowitz, 1990; Tyrrell et al., 1991). K.K.R. was supported by an NSF REU grant and is now at the Center for Visual Science, University of Rochester, Rochester, NY. The authors gratefully acknowledge the assistance of Jeffrey T. Andre, Cathleen M. Daniels, Matthew Factor, David H. Lundy, Philip A. Sanders, Nancy Will, and Lisa Woolslayer for their helpful discussions and assistance in data collection. The authors also thank D. Alfred Owens and Steven W. Spadafore for providing the mirror-moving apparatus and Leonard Matin, D. Alfred Owens, Robert B. Post, and Cynthia A. Spencer for providing critical comments on an earlier version of this manuscript. The contribution of Johannes Dichgans, under the sponsorship of a fellowship from the Alexander von Humboldt Foundation (H.W.L.), is also gratefully acknowledged. Requests for reprints may be sent to either R.A.T. at the Psychology Department, Clemson University, Clemson, SC 29634, or H.W.L. at Psychology Department, Moore Building, The Pennsylvania State University, University Park, PA 16802-3106.
}

visual field as well as the majority of attention and time in this task is devoted to the reading material; the reader locomotes and avoids collisions while taking only intermittent visual samples of the path and obstacles that lie ahead. Although familiarity with the path is not required, observation of this behavior suggests that reading while walking through unfamiliar environments involves a higher frequency of visual sampling than when reading and walking through familiar ones.

Consideration of the demands of either the reading or walking task alone leads to an appreciation for the capacities necessary to perform the two concurrently. It also demonstrates the existence of, and functional difference between, what are now accepted to be two modes of visual processing (Held, 1970; Leibowitz \& Post, 1982). Reading requires recognition vision (the ability to discern details), whereas walking requires guidance vision (the ability to orient spatially).

There is evidence that supports the existence of both functional and anatomical differences between recognition and guidance vision (e.g., Corbetta, Miezin, Dobmeyer, Shulman, \& Petersen, 1991; Farah, 1990; Goodale \& Milner, 1992; Held, 1970; Leibowitz \& Post, 1982; Schneider, 1967; Ungerleider \& Mishkin, 1982; Zeki, Watson, Lueck, Friston, Kennard, \& Frackowiak, 1991). Adequate illumination and some degree of attention are necessary for visual recognition, and the resolving capabilities of the fovea and stimuli with mid-to-high spatial frequencies are necessary for optimal recognition performance. Guidance vision, on the other hand, functions with remarkable insensitivity to both blur and low illumination levels (Banks \& Crowell, 1993; Leibowitz, Post, \& Ginsburg, 1980; Leibowitz, Rodemer, \& Dichgans, 1979; Owens \& Tyrrell, 1993; Tyrrell, Andre, Boonie, Peasley, \& Leibowitz, 1993), and with little or no attention. 
Despite the fact that guidance vision is arguably both phylogenetically older and more critical for survival than recognition vision (locomotion, postural stability, and object localization all rely heavily upon guidance vision), only a small fraction of the research on visual functioning has concentrated on the mechanisms subserving visual guidance (Leibowitz et al., 1980).

In this paper, we describe some of the temporal characteristics of visual guidance. In particular, we hypothesize that information facilitating visual guidance persists and remains useful well beyond the time when the retinal image has faded. Observational support for this hypothesis comes from the knowledge that our ability to locomote is maintained after retinal stimulation is removed, and the knowledge that retinal stimulation is not a necessary condition for the maintenance of space constancy. Further evidence stems from the time course of several illusions of motion. Neither vection (Dichgans \& Brandt, 1978) nor autokinesis (Levy, 1972) occur immediately following stimulus onset. Rather, a substantial latency (generally between 5 and $30 \mathrm{sec}$ ) exists. One possible explanation for the existence of these latencies is that information from the previously viewed environment persists, acts as a stabilizing influence, and delays the onset of the illusion. If this hypothesis is true, then only when the information dissipates beyond a critical level could the body or point of light be perceived to move.

The issue of whether we need continuous visual input for locomotion has received some attention in the field of visuomotor control. In a series of influential experiments, Thomson (1983) measured errors in perceived distance by asking subjects to walk with closed eyes to previously viewed targets that had been placed at distances ranging from 3 to $21 \mathrm{~m}$, and concluded that subjects "internalize" information about "far space." He described this internalized information as being available for only $8 \mathrm{sec}$. After $8 \mathrm{sec}$, Thomson proposed that the information fades severely and suddenly. Other investigators, however, have been unable to replicate Thomson's findings (Elliott, 1986-see Thomson, 1986, for his rebuttal; Rieser, Ashmead, Talor, \& Youngquist, 1990; Steenhuis \& Goodale, 1988). Furthermore, other investigators have found that visual occlusion has relatively little effect on throwing performance (Eby \& Loomis, 1987; Elliott \& Leonard, 1986).

While the debate in the visuomotor literature remains unsettled, investigations into the effects of visual occlusion on perception provide more direct evidence for our hypothesis. Austin, Singer, and Day (1969) seated subjects in front of a tilted room and asked them to adjust a luminous bar so that it appeared to be vertical. Although periods of visual occlusion that separated the exposure and response periods did reduce the expected tilt effect, significant effects were still evident when the occlusion period was as long as $15 \mathrm{~min}$. Similarly, Matin and $\mathrm{Li}$ (1992) reported that exposure to a pitched room induces deviations in visually perceived eye level that persist in darkness. The time constant of the recovery to a more accurate perception of eye level was reported to average approximately $4 \mathrm{~min}$. Thus, the perceptual consequences of exposure to altered visual environments persist for a surprisingly long time.

The theme that emerges from the above investigations is that visual guidance information persists in the absence of retinal stimulation. Data from perceptual and visuomotor experiments, as well as the observation of human performance, suggest that the rate of decay of this information is slow.

Results from an experiment conducted previously in our laboratory have provided further support for our hypothesis (Leibowitz, Tyrrell, Rudolph, \& Eggers, 1993; Tyrrell, Rudolph, Eggers, Li, \& Leibowitz, 1990). In that experiment, 25 subjects viewed a straight path $(34.2 \mathrm{~cm}$ wide $\times 10 \mathrm{~m}$ long) for $10 \mathrm{sec}$. After this exposure, the subjects stood still while their vision was completely occluded for a predetermined occlusion time (OT). OTs ranged from 0 to $240 \mathrm{sec}$ and were not known to the subjects in advance. Following the OT, the subjects attempted to walk within the path at their normal pace. They performed this task quite well with short OTs, but did significantly worse with longer OTs. The performance decrement due to the additional time spent visually occluded occurred gradually and was surprisingly slow; performance had declined only halfway to its minimum value after almost $30 \mathrm{sec}$ of occlusion. Because the effectiveness of the visual exposure to the hallway was adequate initially but decayed over time, these results supported the hypothesis that visual guidance information both persists and gradually decays.

The present experiments support and extend this hypothesis. The experiments provide an independent confirmation of the walking results, test the generality of the phenomenon, and examine the stability of individual differences in performance.

\section{EXPERIMENT 1}

In an effort to provide an independent confirmation that visual guidance information persists, subjects in Experiment 1 performed a visual localization task that had several important differences from the walking task used by Leibowitz et al. (1993). The walking task involved a rich stimulus (the view of a hallway), a complex and coordinated muscular response (walking), and a naturally coupled relationship between the stimulus and the response. The localization task used in this experiment, on the other hand, involved a degraded stimulus (a single point of light), a simple response (thumb movements), and a functionally arbitrary relationship between the stimulus and response.

\section{Method}

Subjects. One hundred forty-three persons (ranging in age from 9 to 69 years with a mean age of 26.8 years), who were attending a scientific exhibit, participated. Each provided informed consent prior to participation and was debriefed afterward.

Apparatus. The apparatus, schematically represented in Figure 1, consisted of a large $(123 \mathrm{~cm}$ high $\times 61 \mathrm{~cm}$ wide $\times 240 \mathrm{~cm}$ deep $)$ light-proof bottomless box mounted on a lower box that was similarly shaped but only $28 \mathrm{~cm}$ high. A 198-cm-long opening in the 


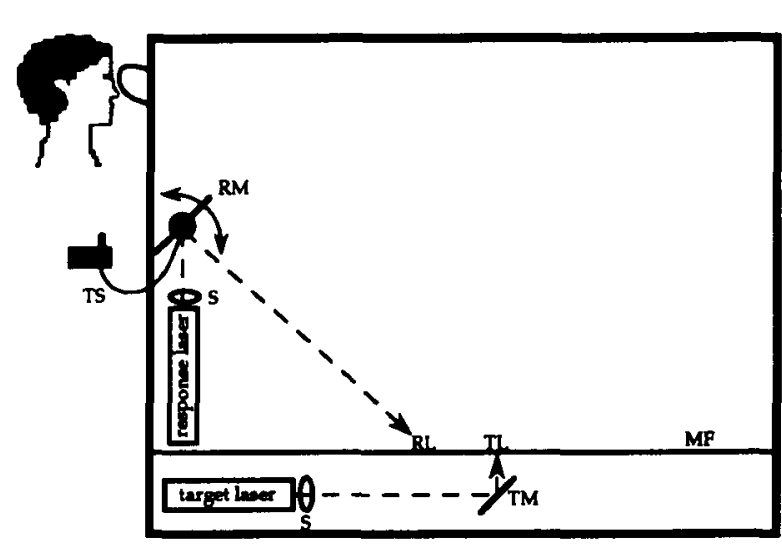

Figure 1. Schematic cross-section of the light-proof apparatus used in the visual localization task (MF = mylar film; $\mathbf{R L}=$ response light; $\mathbf{R M}=$ response mirror mounted on motor; $S=$ shutter; $T L$ $=$ target light; $\mathbf{T M}=$ target mirror; $\mathbf{T S}=$ thumbswitch).

top surface of the lower box was lined with a transparent mylar film to comprise the viewing strip. Goggles mounted on one end of the box provided a binocular viewport. A front-surface mirror reflected the filtered (ND 2.0) beam from a low-power $(0.95 \mathrm{~mW})$ Helium-Neon laser onto the viewing strip to create the target light. This target light was stationary and positioned $40^{\circ}$ beneath eye level so that it bisected the angle defined by the range of motion of the response light as seen from the viewport.

The response light was created by a second, similarly filtered laser, mounted vertically below the viewport. The first-surface mirror that reflected this laser onto the viewing strip was attached to a reversible motor. By operating a handheld thumb switch, the subject could activate the motor and, in doing so, move the response light along the viewing strip in either direction at a speed of approximately $8.0^{\circ}$ of visual angle per second. Microswitches limited the motion of this motor such that the range of motion of the response light was $29.7^{\circ}$ of visual angle. Mechanical shutters mounted on each laser allowed remote control of target presentation. Leftward and rightward movement of the response light was not possible; although the target and response lights were aligned in this axis, the subjects were instructed to ignore any perceived left/right offset between the target and response lights.

Procedure. The subject sat on a stool in front of the apparatus while an experimenter explained that the task would be to manipulate the joystick to move a point of light to the remembered location of a previously viewed point of light. The subject then placed his/her head into the viewport and a thick black cloth was draped over the subject's head to ensure that no light entered the box through the viewport. The subject practiced moving the response light until comfortable with the task.

Although a total of four occlusion times were used $(2,4,16$, and $32 \mathrm{sec}$ ), each subject engaged in only two trials. (A balanced incomplete block design ensured a balanced assignment of OTs to subjects.) Each trial began with a 2-sec presentation of the target light, after which the OT immediately began. During the OT, the subject was free to move his/her eyes but was instructed to keep his/her head in the viewport. Following the OT, the response light was activated, and the subject was instructed to move it to the same position as the target light. The starting position of the response light was always at one of the two endpoints of its range of motion and was counterbalanced across blocks. No time limit was specified, and the subject was free to move the response light back and forth until satisfied. Between measurements, the subject removed his/her head from the viewport.

\section{Results}

Absolute error, defined as the unsigned angular deviation between the positions of the target and response lights, provided the primary index of performance on the localization task. A repeated measures analysis of variance indicated that these errors were significantly larger after longer OTs [Figure $2 ; F(3,270)=6.34, p<.001]$. No other main effects (response light starting position or trial number) or interactions were significant $(p>.05)$. Variable error provided a second index of performance. By combining data across subjects, variable errors (the standard deviation around the mean signed response) index the precision with which the responses were made. As can be seen in Figure 2, these errors also tended to increase following longer OTs.

It is important to remember that the only difference among the four conditions was the duration of the OT, and that the subjects were not aware of the duration of the OT prior to measurement. Thus, the effectiveness of the exposure to the target light diminished during the occlusion period. This is consistent with the hypothesis that visual guidance information persists and gradually decays. However, the essentially linear shape of the functions in Figure 2 was not predicted. This linearity could not continue indefinitely with increasing OTs, and it was expected that the magnitude of the OT effect would be smaller for longer OTs (yielding a decelerating function). It appears that the time course of the localization function is such that OTs of even longer than $32 \mathrm{sec}$ are necessary to determine the asymptote of the function.

\section{EXPERIMENT 2}

To determine the complete time course of the visual localization function, subjects in Experiment 2 performed

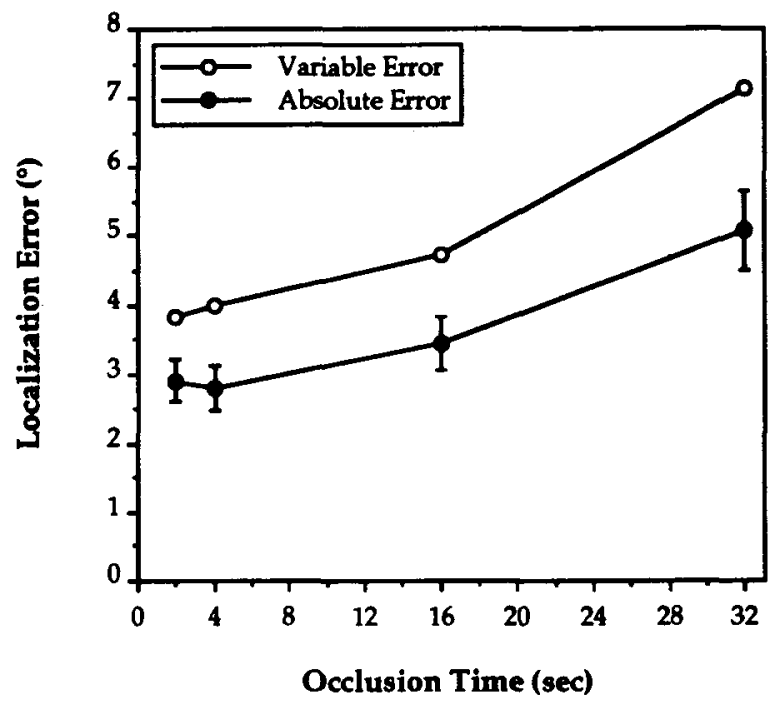

Figure 2. Mean ( $\pm 1 S E$ ) absolute error and mean variable error in localizing a previously viewed point of light after various durations of visual occlusion. 
the task with a wider range of OTs. To compare the effects of visual occlusion on localization and walking performance, the subjects also performed the walking task that was used by Leibowitz et al. (1993). If the effects reported in Experiment 1 and by Leibowitz et al. (1993) were due to the common mechanism of visual guidance information persisting and slowly decaying, then one would expect there to be a relationship between performance on the two tasks.

\section{Method \\ Subjects. Twenty-eight students (ranging in age from 16 to 26 years with a mean age of 20.7 years) volunteered to participate. Each provided informed consent prior to participation and received verbal and written debriefings following the experiment. When asked, they all denied that they had any medical condition or in- jury that could potentially interfere with their ability to perform the walking task.}

Apparatus. The localization apparatus described in Experiment 1 was used. The walking task was conducted in a long open hallway. Two strips of black tape were applied to a white floor with a separation of $34.2 \mathrm{~cm}$ to define a straight $10.0-\mathrm{m}$-long path.

Procedure. Half of the the subjects performed the walking task first and the other half performed the localization task first. Within each task, two trials at each of seven OTs $(2,4,8,16,32,64$, and $128 \mathrm{sec}$ ) were tested in a random order. In both tasks, the subject was aware of the range of OTs, but not the OT of any given trial.

The procedure used for the localization task was identical to that in Experiment 1, with the exception that the subject was told to keep his/her head in the viewport between trials while an opaque screen occluded the view of the interior of the apparatus.

Before the walking task, the subject was told that the task was to walk blindfolded at a normal pace as far as possible within the path. The subject was assured that he/she would be stopped by an experimenter before making contact with a wall. Prior to beginning the actual experiment, the subject walked the path twicefirst with normal visual feedback, and then while wearing a pair of goggles that completely occluded vision. This procedure established the subject's normal walking pace and helped familiarize the subject with the task.

At the start of each trial, the subject raised the goggles to rest on his/her forehead, positioned his/her feet behind a starting line, and aimed his/her body toward the path. After viewing the path for $10 \mathrm{sec}$, the subject was told to lower the goggles. The subject then stood still during the OT. Following the OT, a tone sounded and the subject was instructed to "begin walking." Two experimenters followed the subject down the path. One experimenter measured the distance that the subject walked within the path-the distance from the starting line to the point at which any part of either of the subject's feet first landed outside the path. The second experimenter served as a "spotter" and stopped the subject before contact was made with a wall. In order to minimize feedback, the subject was allowed to continue walking outside the path until a wall was approached. At that point, the second experimenter led the subject back to the starting point while the subject's vision remained occluded. Between trials, the subject was reminded to maintain a normal walking pace until told to stop. Performance was coded as $10.0 \mathrm{~m}$ whenever the subject succeeded in walking the entire distance of the path without stepping outside the boundaries.

\section{Results}

As in Experiment 1, absolute error in the localization task was significantly greater after longer OTs [Figure 3;
$F(6,162)=12.45, p<.0001]$. Also similar to the data from Experiment 1 , the increase in absolute error was essentially linear with OTs less than $32 \mathrm{sec}$. With OTs greater than $32 \mathrm{sec}$, however, the function decelerates, resulting in the curvilinear form that was anticipated. This same form can be seen in the function that relates variable error to OT.

The subjects were less able to walk in the direction of the path after longer OTs $[F(6,144)=6.46, p<.0001]$. This result, depicted in Figure 3, supports the hypothesis that the visual guidance information that was available during the 10-sec exposure period persisted and was initially useful, but gradually decayed.

The functions in Figure 3 follow a remarkably similar time course. However, the "half-lives" of the functions (the duration of occlusion that would correspond to the performance level midway between the maximum and minimum measured levels) are not identical: While performance on the localization task was halved after slightly more than $16 \mathrm{sec}$, the half-life of the walking function was greater than $30 \mathrm{sec}$. This difference is due to the steeper initial drop in localization performance relative to the initial rate of decrease in walking performance.

Two techniques were used to examine the relationship between performance on the two tasks. A significant correlation $[r(194)=-.21, p=.003]$ between performance on the walking task and absolute error in the localization task (using each subject's average absolute error after each OT) indicated that when the subjects performed well on one task (i.e., a relatively long distance walked), they also tended to perform well on the other (i.e., a relatively small absolute error). ${ }^{1}$ To determine whether individual differences in the performance decrements on the two tasks are related, a score was derived to provide, for each task, a single measure of each subject's OT-related performance decrement (with absolute error indexing localization performance). For each subject and task, performance was averaged across the three longest OTs $(32,64$, and $128 \mathrm{sec})$ and was subtracted from the performance averaged across the three shortest OTs $(2,4$, and $8 \mathrm{sec})$. As expected, these difference scores were significantly different from zero [mean walking difference $=153.2 \mathrm{~cm} ; t(26)=5.43, p<$ .0001 ; mean absolute error difference $=-2.3^{\circ} ; t(26)=$ $-8.85, p<.0001]$. The correlation between these scores was not significant $(r=.23, p>.10)$.

Thus, the effect of visual occlusion on localization and walking performance successfully replicated the effects reported in Experiment 1 and by Leibowitz et al. (1993), respectively. Although absolute performance was related across the two tasks, there appears to be no simple relationship between the performance decrements.

\section{EXPERIMENT 3}

To determine the extent to which the influence of visual occlusion on performance is a stable individual charac- 


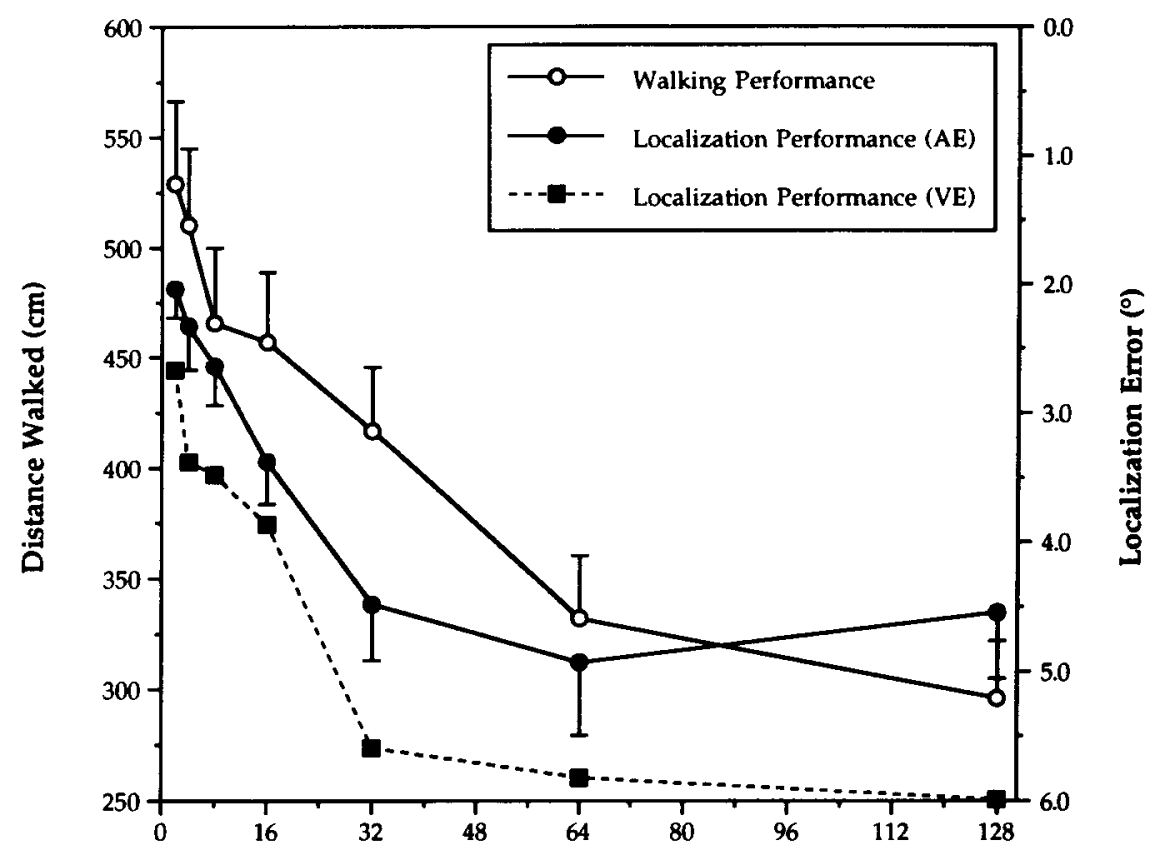

Occlusion Time (sec)

Figure 3. Mean walking performance $(+1 S E)$, mean absolute localization error $(\mathrm{AE} ;+1$ $S E$ ), and mean variable localization error (VE) after various durations of visual occlusion. On each task, performance decreased with greater occlusion time. These performance decrements followed a similar time course. The $y$-axis of the localization functions has been inverted to facilitate this comparison.

teristic, subjects performed the localization and walking tasks on multiple occasions.

\section{Method}

Subjects. Twenty-one students (ranging in age from 17 to 22 years with a mean age of 19.6 years) participated. Most received credit for an introductory psychology class in exchange for participating, and none reported having any medical condition or injury that could affect performance. Verbal and written debriefings were provided after the last session was completed.

Apparatus. The localization apparatus and walking pathway were identical to those described previously.

Procedure. Each subject participated in both tasks on three separate occasions. The initial "practice" session familiarized the subject with the experimental protocol and reduced the effect of practice between the following two experimental sessions. Although the data collected during the practice session are not reported, the procedures followed in all three sessions were identical.

The order in which the two tasks were performed was constant across sessions for each subject. Eleven subjects performed the walking task first, and 10 performed the localization task first. A minimum of $24 \mathrm{~h}$ separated the practice session from the first experimental session. The separation between the two experimental sessions averaged $22.9( \pm 3.8)$ days.

Because the purpose of this experiment was to examine the stability of the OT effect and not to replicate the shape of the functions, performance was examined after only two OTs (5 and $60 \mathrm{sec}$ ). The average difference in performance between the two OTs provided a measure of the magnitude of the OT effect. During each session and for each task, the subjects performed three trials at both of these OTs. To avoid having predictable OTs, they also performed one trial each at OTs of 10,45 , and $90 \mathrm{sec}$. (The data from these trials are not reported.) This ensured that after $5 \mathrm{sec}$ of occlusion, the subject would not be able to deduce that the trial was to involve $60 \mathrm{sec}$ of occlusion. The order of the nine trials was determined randomly for each subject, session, and task.

\section{Results}

Absolute errors on the localization task were significantly higher after the longer OT [paired $t(20)=2.69$, $p=.014]$. This was also true for the variable errors around the mean of the three responses that each subject performed at each OT [paired $t(20)=3.21, p=.002$ ]. The OT effect in the walking task also reached significance [paired $t(20)=2.60, p=.017$ ]. Once again, performance on both tasks was worse after the longer OT.

The mean magnitude of the effect of OT on absolute errors in the first localization session was significantly correlated with the mean magnitude of the OT effect in the second localization session $(r=.50, p=.021)$. This finding, depicted in the top of Figure 4, indicates that those subjects whose ability to position the response light near the position of the target light was severely degraded during the extra $55 \mathrm{sec}$ of OT in the first session tended to be similarly affected by the extra OT in the second session. That is, over a period of approximately 3 weeks, the effect of visual occlusion on absolute error was consistently large for some individuals and consistently small for others. When examined from the perspective of the 
Localization Task

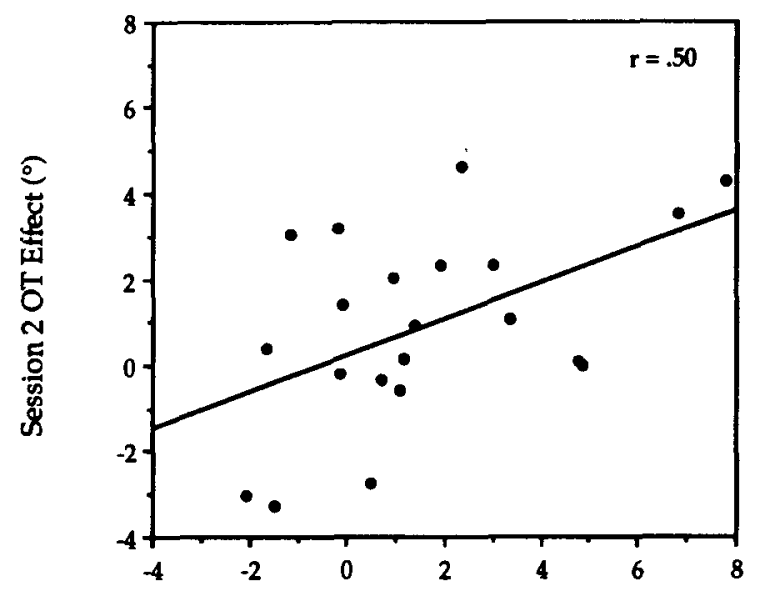

Session 1 OT Effect $\left({ }^{\circ}\right)$

Walking Task

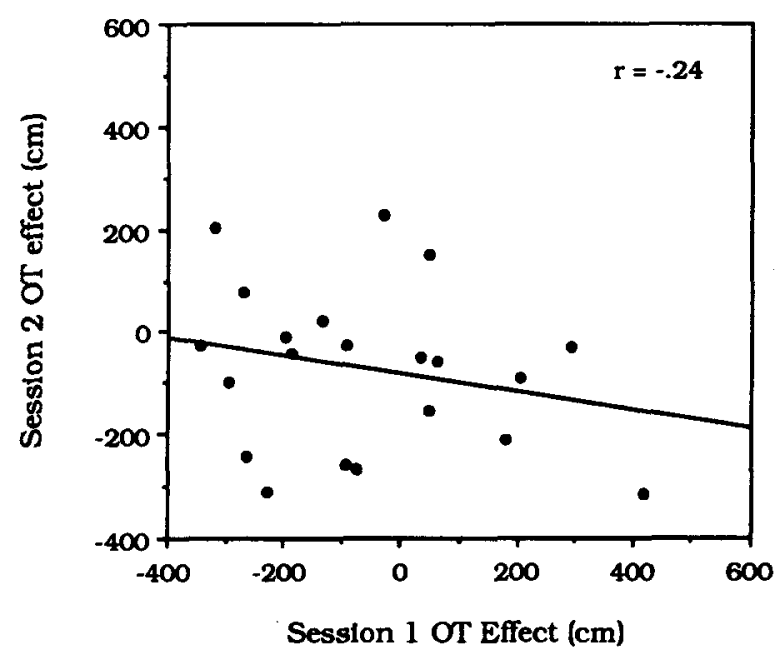

Figure 4. Scatterplot illustrating the relationship between the OTrelated performance decrement across the two experimental sessions for absolute errors in the localization task (upper graph) and for the walking task (lower graph). For each task, the performance decrement is defined as the mean performance difference between the two OTs (performance after 60-sec OT minus performance after 5-sec OT).

hypothesis, this result suggests that the rate at which the effectiveness of visual guidance information decreases is a stable individual characteristic. The effect of OT on variable error, however, was not correlated across sessions $(r=.10, p>.10)$

The magnitude of the OT effect in the first walking session was not correlated with that in the second walking session $(r=-.24, p>.10$; see bottom of Figure 4$)$. Therefore, knowledge of the effect of OT on an individual's walking performance in the first session is not useful in predicting the same subject's OT effect in the second session.

\section{DISCUSSION}

The tasks used in these experiments have vast differences. Although the walking task involves a rich stimulus, a complex and coordinated motoric response, and a natural coupling between the stimulus and the response, the localization task involves a simple stimulus that is arbitrarily related to a simple response. Furthermore, postural stability (which is normally partially controlled by visual mechanisms) is a component of the walking task but not the localization task. Despite these differences, an important pattern emerged that is common to both tasks. On neither task was performance impossible immediately following visual occlusion. Rather, the subjects were able to perform quite well after short OTs. With longer OTs, however, consistent and significant performance decrements were evident. Given the differences between the tasks, there is a surprising similarity in the rate at which performance on the tasks deteriorated (Figure 3). These results support the hypothesis that visual guidance information persists, and then slowly decays, in the absence of retinal stimulation.

Although this hypothesis is similar to Thomson's (1983) proposal that information about the environment is available for up to $8 \mathrm{sec}$, our data indicate that visual guidance information persistence follows a time course that is even longer (a suggestion that was also made by Steenhuis \& Goodale, 1988) and degrades gradually (as compared with the sharp cutoff proposed by Thomson). The long time course (see Figure 3 ) rules out the possibility that iconic memory alone limits performance on these tasks, because the time courses of the present data are approximately two orders of magnitude longer than that of iconic memory (e.g., Loftus, Johnson, \& Shimamura, 1985).

In addition to the differences between the tasks that have already been mentioned, it was found in Experiment 3 that only the localization task exhibited a degree of stability in the magnitude of the occlusion effect over a 3-week period. Given these many differences, it is not completely surprising that there was no simple relationship between the performance decrements of the tasks. The correlation between the absolute performance levels suggests, however, that there is some degree of intertask consistency in the magnitude of the effect of occlusion for a given individual.

The ability to perform visually mediated tasks when visual exposure is both brief and substantially separated in time from the behavior has significant biological utility. Persistence of visual guidance information facilitates space constancy by reducing the need to continually monitor the environment that we interact with and locomote through. The same persistence frees us to attend to other tasks while we locomote and enables us to interact with an environment that was seen moments ago. For instance, it enables us to maintain our position on the roadway as we glance at the instruments inside our vehicle or when our view of the roadway becomes temporarily obstructed (Senders, Kristofferson, Levison, Dietrich, \& Ward, 
1967). It allows predators to track their prey visually as they move over a varied terrain and allows the prey to monitor the position of their predator while fleeing.

The persistence of visual guidance information also provides a framework from which to examine the temporal characteristics of several other visually mediated phenomena. Individual differences in the rate of decay provide a testable hypothesis for explaining the individual differences in the latencies of autokinesis (Levy, 1972), vection (Dichgans \& Brandt, 1978), and height vertigo (T. Brandt, 1989 , personal communication), and in the onset of body sway in darkness (G. Stelmach, 1989, personal communication). Furthermore, when viewed from the current perspective, the slow perceptual recovery from altered visual environments reported by Austin et al. (1969) and Matin and $\mathrm{Li}$ (1992) is not surprising. Although the subjects in those experiments gave their responses while in darkness, their responses were based on information that had persisted from their preceding visual experience.

The persistence phenomenon may also be useful in explaining, diagnosing, and predicting problems related to spatial orientation. Task performance during visual occlusion may be useful in identifying individuals likely to suffer from "disorientation." For example, falls are a major health problem for the elderly (Leibowitz \& Shupert, 1985). If the visual mechanisms that facilitate postural stability (e.g., Simoneau, Cavanagh, Ulbrecht, Leibowitz, \& Tyrrell, 1991; Simoneau, Leibowitz, Ulbrecht, Tyrrell, \& Cavanagh, 1992) are subserved by persisting visual guidance information, then tests that index this persistence may aid in identifying individuals who are at risk for falls. Additionally, patients with Parkinson's disease often have difficulty in orienting to recently viewed scenes (J. Dichgans, 1990, personal communication). That many of these patients experience difficulty in sitting down after having turned their backs on their chairs or in traversing a ground surface with reduced visual contours suggests that a symptom of this disease is that visual guidance information decays more rapidly.

Although the phenomenon of persistence does have tremendous biological utility, it is possible that the persistence of information from previously viewed scenes may be detrimental for performance in some situations. Whenever our visual environment changes rapidly, there is the potential for the information from the previous environment to interfere with the new information. For example, the onset of disorientation problems related to the landing of single-pilot aircraft often coincides with the moment that pilots emerge from cloud cover and make a rapid visual transition from the cockpit to the landing strip (Leibowitz, in press). This may be due to visual information from the cockpit persisting and interfering with the information being obtained from the outside environment.

The persistence of visual guidance information provides still another functional difference between the processes subserving visual recognition and visual guidance. It is clear from both the present experiments and the phenomena described above that the decay process is both slow and gradual. The persistence of visual guidance information is consistent with a wide range of behaviors involving spatial orientation. The implications of this phenomenon will depend ultimately on the relation between individual differences in persistence and variables such as age, training, familiarity with the environment, and neurological health. Given the accessibility of the construct and the wide range of behaviors for which visual guidance information is critical, such data would lead to a more complete understanding of visual functioning.

\section{REFERENCES}

Austin, M., Singer, G., \& DAY, R. H. (1969), Visual orientation illusion following judgments with a tilted visual field. Nature, 221, 583-584.

BanKs, M., \& Crowell, J. (1993, January). The optic flow information for safe driving. Paper presented at the 1993 meeting of the Transportation Research Board, Washington, D.C.

Corbetta, M., Miezin, F. M., Dobmeyer, S., Shulman, G. L., \& PETERSEN, S. E. (1991). Selective and divided attention during visual discriminations of shape, color, and speed: Functional anatomy by positron emission tomography. Joumal of Neuroscience, 11, 2383-2402.

Dichgans, J., \& BrandT, T. (1978). Visual-vestibular interaction: Effects on self-motion perception and postural control. In R. Held, H. Leibowitz, \& H. L. Teuber (Eds.), Handbook of Sensory Physiology (Vol. 8, pp. 755-804). Berlin: Springer-Verlag.

EBY, D. W., \& Loomis, J. M. (1987). A study of visually directed throwing in the presence of multiple distance cues. Perception \& Psychophysics, 41, 308-312.

ElLiotT, D. (1986). Continuous visual information may be important after all: A failure to replicate Thomson. Joumal of Experimental Psychology: Human Perception \& Performance, 12, 388-391.

ElliotT, D., \& LEONARD, K. (1986). Influence of a no-vision delay on throwing accuracy. Perceptual \& Motor Skills, 63, 517-518.

FARAH, M. J. (1990). Visual agnosia: Disorders of object recognition and what they tell us about normal vision. Cambridge, MA: MIT Press.

GoOdAlE, M. A., \& MiLnER, A. D. (1992). Separate visual pathways for perception and action. Trends in Neuroscience, 15, 20-25.

HeL.D, R. (1970). Two modes of processing spatially distributed visual stimulation. In F. O. Schmitt (Ed.), The neurosciences: Second study program (pp. 317-324). New York: Rockefeller University Press.

Leibowitz, H. W. (in press). Some basic mechanisms in spatial orientation. Proceedings of Air Force Conference on Spatial Disorientation, Bolling Air Force Base.

Leibowitz, H. W., Post, R. B. (1982). The two modes of processing concept and some implications. In J. Beck (Ed.), Organization and representation in perception (pp. 343-363). Hillsdale, $\mathrm{NJ}$ : Erlbaum.

Leibowitz, H., Post, R., \& Ginsburg, A. (1980). The role of fine detail in visually controlled behavior. Investigative Ophthalmology \& Visual Science, 19, 846-848.

Leibowitz, H. W., Rodemer, C. S., \& Dichgans, J. (1979). The independence of dynamic spatial orientation from luminance and refractive error. Perception \& Psychophysics, 25, 75-79.

LEIBOWITZ, H. W., \& SHUPERT, C. L. (1985). Spatial orientation mechanisms and their implications for falls. Clinics in Geriatric Medicine, $1,571-579$.

Leibowitz, H. W., Tyrrell, R. A., Rudolph, K. K., \& Eggers, B. G. (1993). The persistence of visual guidance information: An additional distinction between recognition and guidance vision. Manuscript in preparation.

LEVY, J. (1972). Autokinetic illusion: A systematic review of theories, measures, and independent variables. Psychological Bulletin, 78, 457-474.

Loftus, G. R., Johnson, C. A., \& Shimamura, A. P. (1985). How much is an icon worth? Journal of Experimental Psychology: Human Perception \& Performance, 11, 1-13. 
MAtin, L., \& LI, W. (1992). Mislocalizations of visual elevation and visual vertical induced by visual pitch: The great circle model. In B. Cohen, D. Tomko, \& F. Guedry (Eds.), Symposium on sensing and controlling motion: Vestibular and sensorimotor function ( $\mathrm{pp} .242-$ 265). Annals of the New York Academy of Sciences, 656, 242-265.

OWens, D. A., \& TYRRELL, R. A. (1993). Visual guidance performance of young and older drivers in challenging conditions. Investigative Ophthalmology \& Visual Science, 34, 1418.

Rieser, J. J., Ashmead, D. H., Talor, C. R., \& Youngquist, G. A. (1990). Visual perception and the guidance of locomotion without vision to previously seen targets. Perception, 19, 675-689.

SCHNEIDER, G. E. (1967). Contrasting visuomotor functions of tectum and cortex in the golden hamster. Psychologische Forschung, 31, $52-62$.

Senders, J. W., Kristofferson, A. B., Levison, W. H., Dietrich, C. W., \& WARD, J. L. (1967). The attentional demand of automobile driving. Highway Research Record, 195, 15-32.

Simoneau, G. G., Cavanagh, P. R., Ulbrecht, J. S., Leibowitz, H. W., \& TYRrell, R. A. (1991). The influence of visual factors on fall-related kinematic variables during stair descent by older women. Journal of Gerontology: Medical Sciences, 46, M188-M195.

Simoneau, G. G., Leibowitz, H. W., Ulbrecht, J. S., Tyrrell, R. A., \& Cavanagh, P. R. (1992). The effects of visual factors and head orientation on postural steadiness in women 55-70 years of age. Journal of Gerontology: Medical Sciences, 47, M151-158.

Steenhuis, R. E., \& Goodale, M. A. (1988). The effects of time and distance on accuracy of target-directed locomotion: Does an accurate short-term memory for spatial location exist? Joumal of Motor Behavior, 20, 399-415.

Tномson, J. A. (1983). Is continuous visual monitoring necessary in visually guided locomotion? Journal of Experimental Psychology: Human Perception \& Performance, 9, 427-443.

THOMson, J. A. (1986). Intermittent versus continuous visual control: A reply to Elliott. Journal of Experimental Psychology: Human Perception \& Performance, 12, 392-393.
Tyrrell, R. A., Andre, J. T., Boonie, K. K., Peasley, C. E., \& Leibowitz, H. W. (1993). On the visual information that supports blind walking. Investigative Ophthalmology \& Visual Science, 34, 1418.

Tyrrell, R. A., Daniels, C. M., Andre, J. T., Lundy, D. H., EgGERS, B. G., \& LEIBOwITZ, H. W. (1991). The effects of time and eye movements on pointing performance. Investigative Ophthalmology \& Visual Science, 32, 1272.

Tyrrell, R. A., RudolPh, K. K., Eggers, B. G., Li, Y., \& Leibowitz, H. W. (1990). Do ambient orientation cues persist in the absence of visual stimulation? Investigative Ophthalmology \& Visual Science, 31(Suppl.), 327.

UNGERLEIDER, L. G., \& MiShKIN, M. (1982). Two cortical visual systems. In D. J. Ingle, M. A. Goodale, \& R. J. W. Mansfield (Eds.), Analysis of visual behavior (pp. 549-586). Cambridge, MA: MIT Press.

Zeki, S., Watson, J. D. G., Lueck, C. J., Friston, K. J., Kennard, C., \& FraCKowIAK, R. S. J. (1991). A direct demonstration of functional specialization in human visual cortex. Journal of Neuroscience, 11, 641-649.

\section{NOTE}

1. This intertask correlation is unchanged when between-subject variability is reduced by "centering" the data (subtracting each subject's overall mean from his/her values at each OT). It appears that the withinsubject variations are responsible for the relationship between the two tasks: Conditions that led a subject to perform well on one task also led to a high level of performance on the other task.

(Manuscript received January 27, 1992; revision accepted for publication March 23, 1993.) 